REVIEW

\title{
Fit to fly: practical challenges in neonatal transfers by air
}

\author{
C H Skeoch, L Jackson, A M Wilson, P Booth
}

Arch Dis Child Fetal Neonatal Ed 2005;90:F456-F460. doi: 10.1136/adc.2004.064949

Air transport of infants in incubators should be undertaken in a manner that is safe for both staff and infant and satisfies all directives by appropriate regulatory bodies. In Scotland during the last two years, certification of an infant incubator system for use in both rotary and fixed wing aircraft has been accomplished. This is a report of the issues addressed during this project, which will be common to all neonatal transport services intending to develop air transport capability. of NeTS and the experience of the SAS Air Wing, it became increasingly clear that substantial change was necessary to comply with regulatory agencies, particularly with respect to the weight of the transport incubator system (TIS) and predefined loading and unloading procedures. With the centralisation of specialist Scottish services, especially cardiac surgery and extracorporeal life support, infants with more complex clinical problems required more frequent urgent transfer over greater geographical distances. National integration was required for the organisation of safe air transport for such clinical scenarios. These changes are now discussed.

\section{DEVELOPMENT OF EQUIPMENT FOR ROAD/AIR INTEGRATED USE}

The development of an air transport service should not be undertaken in isolation from issues pertaining to the use of road ambulances. At the outset a project leader was identified who had the required background knowledge and inter-professional links required to integrate clinicians, aviation operators, ambulance managers, engineers, and other disciplines. Meeting together at an early stage ensured that the aims of the project were met within an agreed time scale and to the reasonable satisfaction of all interested parties. From the outset it was clear that compromises would have to be accepted by all participating groups, including clinicians.

Multiple factors influenced and mandated the course of the service development. Any group commissioning an air transport service for the newborn should consider these.

\section{REGULATORY BODIES \\ European aviation safety agency (EASA; http://www.easa.eu.int/home/ index.html)}

This agency has replaced the Civil Aviation Authority, which previously regulated the adaptation of transport incubators for aircraft use. The section pertaining to this is part 21 . In summary, before a TIS can be placed in an aircraft for flight, EASA must be happy that every component part of that system is tested within the environment of the aircraft. To achieve compliance, clinicians must coordinate with an aviation operator and an appropriately certified engineering and production firm. We undertook this project with Bond Air Services, SAS, and Paraid Limited. These, and other such

Abbreviations: CEN, European committee for standardisation; EASA, European aviation safety agency; iNO, inhaled nitric oxide; NeTS, neonatal transport service; SAS, Scottish Ambulance Service; TIS, transport incubator system 


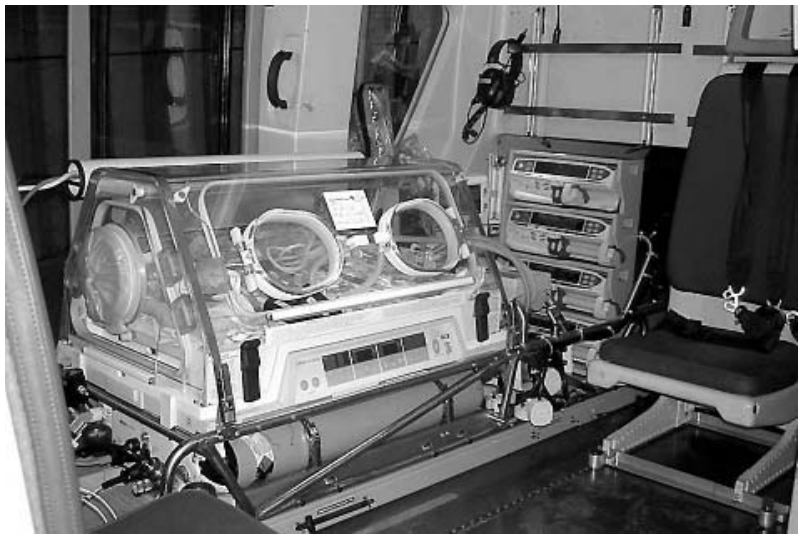

Figure 1 Cage assembly designed and developed to allow all component parts of the transport incubator system to be adequately placed for clinician use and suitably secured to satisfy the European aviation safety agency regulations.

appropriately certified companies, will lead clinicians through the maze of technical requirements culminating in EASA certification.

Health and safety executive (http://www.hse.gov.uk) This governmental organisation is responsible for ensuring the health and safety of the team who transport the infant. Legislation covers diverse issues including noise, vibration, exposure to noxious substances, and manual handling.

\section{European committee for standardisation (CEN; http://} www.cenorm.be/cenorm/index.htm)

Individual components on transport incubators that are normally used in the hospital setting are CEN compliant. There are draft regulations on transport incubator trolleys for road vehicles awaiting ratification. It is accepted that some road vehicles used to transport neonates are not CEN compliant.

\section{TECHNICAL ISSUES AFFECTING DESIGN AND BUILD OF TIS \\ Top frame assembly}

To allow all component parts of the TIS to be adequately placed for clinician use and suitably secured to satisfy the EASA regulations, a new cage assembly had to be designed and developed (fig 1). An electrical installation report was necessary, examining the TIS batteries and external power sources. The electromagnetic current signature of the whole

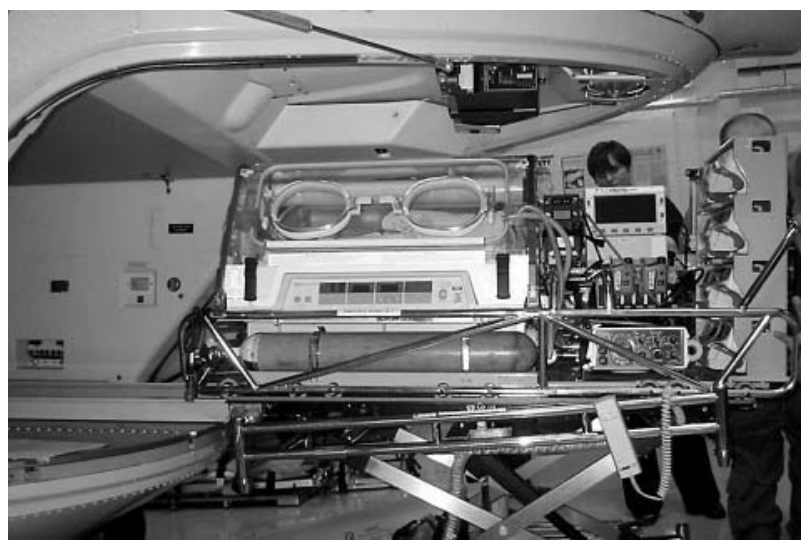

Figure 2 Top frame assembly locked into the starboard side of the aircraft. installation in both aircraft was examined. Consideration of these issues related to the aircraft avionics, radio and navigation equipment, and fuel computers. Electrical integrity of the TIS included testing for stability in the event of a power surge during flight. It is important to realise that medical equipment that receives certification is specific for that exact model and a specific aircraft. Any change, no matter how minor, invalidates the certification.

Detailed stress reports were commissioned to establish the integrity of the top frame assembly in the event of rapid deceleration or acceleration. Different $G$ force stability requirements pertain to fixed wing, rotary wing, and land based vehicles, therefore an "envelope" G force requirement was adapted. This stipulated integrity to $10 \mathrm{G}$ backwards, up, down, and sideways, $16 \mathrm{G}$ in a forward direction, and $2 \mathrm{G}$ rearwards.

Stress reports had to cover the areas of medical equipment security, the incubator assembly itself, the attachment of the loading guide to the aircraft, and the security of batteries and cylinders to the top frame assembly. Significant modifications were required to the TIS to ensure certification including design and manufacture of a holding system for the syringe pumps and a method of securing the TIS canopy. The weight of the TIS, including the trolley interface and all equipment, was to be kept to below $140 \mathrm{~kg}$.

\section{Loading and unloading}

A loading guide system for the Eurocopter 135 was developed which the helicopter engineers can fit in 30 minutes. The TIS has an electrically activated raising and lowering mechanism. The top frame assembly separates from the trolley and glides into the helicopter on a wheel based system, securely locking into the starboard side of the aircraft (fig 2). Power and medical gas supply comes from the helicopter. The trolley base is then loaded in the aft of the helicopter (fig 3).

The same TIS is used in the Beech King Air 200, the only difference being that the Lifeport Patient Loading Utility System (LifePort Inc, Woodland, Washington, USA) is used, which also provides power and medical gas supply to the TIS.

Great effort must be made to ensure that all support systems in the aircraft are specifically adapted for use for infants and the TIS. It is essential to ensure correct length and connections for electrical leads and gas hoses, and appropriate specification for all equipment. This particular problem regarding common DC leads and plugs has been addressed by a helpful document from the New South Wales transport team (www.isas.org.au/main/medpower.htm). Several dry runs with the incubator in the aircraft under full and extreme operational conditions are necessary to identify and solve such problems.

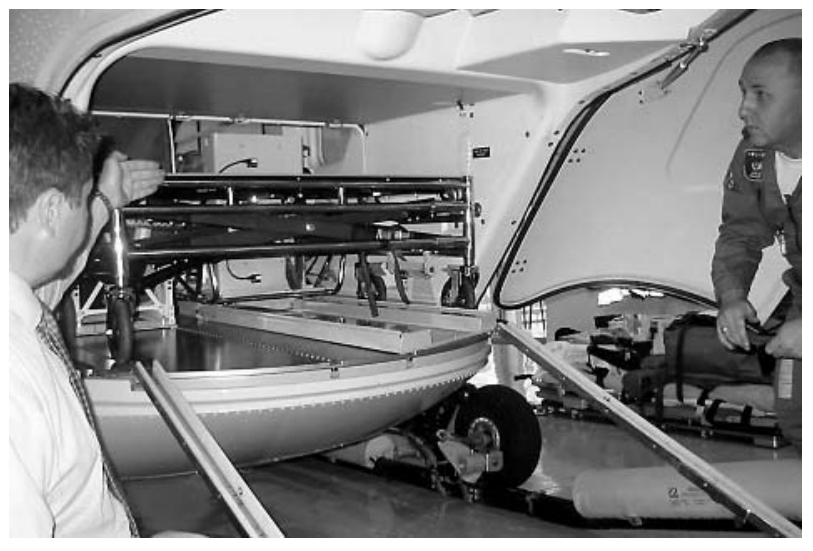

Figure 3 Trolley base loaded in the aft of the helicopter. 


\section{ARRANGING A TRANSPORT}

Lack of an integrated air and road transport service will lead to chaos. The SAS have been pioneering their approach to this, and we have worked closely with them to ensure smooth and seamless communication. We have had to adopt a number of rules to achieve these aims.

- There is one dedicated telephone number for the SAS air desk, which is used by any clinician to initiate an air transfer.

- Once a transfer has been accepted, the lead transport team member contacts the air desk to arrange the transport.

- After discussions with the National Neonatal Transport Executive and senior members of the SAS, a matrix has been agreed whereby known journeys across and outwith Scotland have been designated as requiring air transport.

- Transfers of non-emergency patients such as "back transfers" may be undertaken by air but only after prior discussion with the SAS.

- In addition to the agreed matrix, other factors are taken into consideration such as distance, duration of journey, availability of aircraft, and weather conditions.

- The Air Wing will arrange road ambulances to take the transport team to the aircraft and also deliver the transport team plus the infant from the aircraft to the receiving hospital. They ensure ambulance/TIS compatibility. In this fashion one phone call from the transport team initiates an integrated service response from the SAS, across health board, national, and international boundaries.

\section{AIRCRAFT AVAILABILITY}

The SAS has undergone a reprocurement exercise based on a number of factors. This has led to the intent to have appropriate aircraft positioned throughout Scotland reflecting the highest frequency of clinical use. It was important to meet with adult and paediatric intensivists to understand their needs, make compromises where necessary, and develop a hierarchical system for use of assets. If necessary, the air desk can arrange the use of Ministry of Defence or Coastguard helicopters. There is a signed agreement, however, that on each occasion this request is made, that all alternative means of transport have been explored and that the situation is life or death. It has to be remembered that the primary function of these military assets are for search and rescue of other military assets and not as an air ambulance service.

\section{PROTOCOLS}

The normal air transfer involves six loading/unloading events. Much of the transfer is carried out in the aircraft where it is simply not possible to stop immediately. The environment may be hostile. Under these circumstances it is vital that clinical protocols are used to prepare or "package" the infant. It is equally important to have guidelines for adverse clinical events during flight. Much of these may be minimised by taking into account the effect of the flight environment and the physiological effects of that on the pathology affecting the infant. ${ }^{23}$ Communication issues should be addressed to ensure adequate inter-team and air to ground links at all times. It should be noted that flying helmets with integral communication links may require microphones of differing impedances depending on the type of helicopter in use. We have solved this problem using a system supplied by Headset Services Ltd (www.headsetservices. com).

\section{ALTITUDE}

Personnel undertaking air transfer must have an understanding of the potential effect of altitude on the infant's clinical status. Total barometric pressure decreases with altitude. At sea level, barometric pressure is $760 \mathrm{~mm} \mathrm{Hg}$ compared with $565 \mathrm{~mm} \mathrm{Hg}$ in a standard commercial airline leading to an equivalent percentage reduction in partial pressure of oxygen. Every effort therefore must be made to maximise oxygen delivery in hypoxic infants by other means before an air transfer by maintaining an adequate systemic blood pressure and haemoglobin concentration. ${ }^{4}$

Air expands at increasing altitude, and it is important to realise that under normal conditions a pressurised aircraft maintains a cabin altitude equivalent to about $2500 \mathrm{~m}$. Therefore it is quite possible under these circumstances for an innocuous air leak at sea level to expand and become clinically significant. Every effort must therefore be made to ensure that any trapped air liable to expand at altitude is effectively drained. This includes apparently trivial pneumothoraces, which, in a controlled environment of a neonatal unit, may not require draining. Our protocol dictates that infants who may be liable to air leak, such as those with severe meconium aspiration syndrome, should be transferred with the cabin altitude set to sea level. The Eurocopter 135 is unpressurised but with adequate pretransfer stabilisation offers few disadvantages in terms of altitude associated problems. The decision about whether to use rotary or fixed wing aircraft is rather made on the basis of distance, weather, and available landing sites.

\section{TAKE OFF AND LANDING}

Rapid acceleration during take off, with the infant secured head forward, theoretically results in reduced cerebral perfusion. Conversely, on landing, rapid deceleration may cause a sudden rise in venous cerebral perfusion. There is provisional evidence that premature infants undergoing transfer may have a higher incidence of intraventricular bleeding. ${ }^{5}{ }^{6}$ However, the clinical effect of these controllable events requires clarification.

\section{THERMAL ISSUES}

The importance of maintaining an appropriate thermal environment for the neonate and the avoidance of cold stress has been well documented. ${ }^{78}$ There is a temperature drop of $2^{\circ} \mathrm{C}$ for every $300 \mathrm{~m}$ of altitude, and in unheated military helicopters this may put high demands on the TIS. Such demands on battery power require consideration. A reliable method of measuring infant temperature during transport must be used. Reducing heat loss and conservation of battery power on the TIS include use of Isocovers (Eden Medical, Loanhead, Midlothian, Scotland, UK), Transwarmer mattresses (Draeger Medical, Lubeck, Germany), bubble wrap, and hats. TIS used for air transport must always have fully charged batteries at the beginning of a transfer. DC power cables suitable for both the aircraft and the ambulance should be taken. Strong sunlight can also cause problems with incubator overheating, and incubator covers are useful in this situation.

\section{NOISE AND VIBRATION}

Vibration is not usually detrimental to the infant, but can dislodge lines and tubes and adversely effect monitoring equipment. ${ }^{9}$ Consideration should be given to equipment specifically designed to minimise the effect of movement artefact such as pulse oximetry using Masimo or Oxismart technology. During transport all lines should be secure and visible, particularly arterial lines, to allow observation without the need to open the incubator. Squishon gel filled 


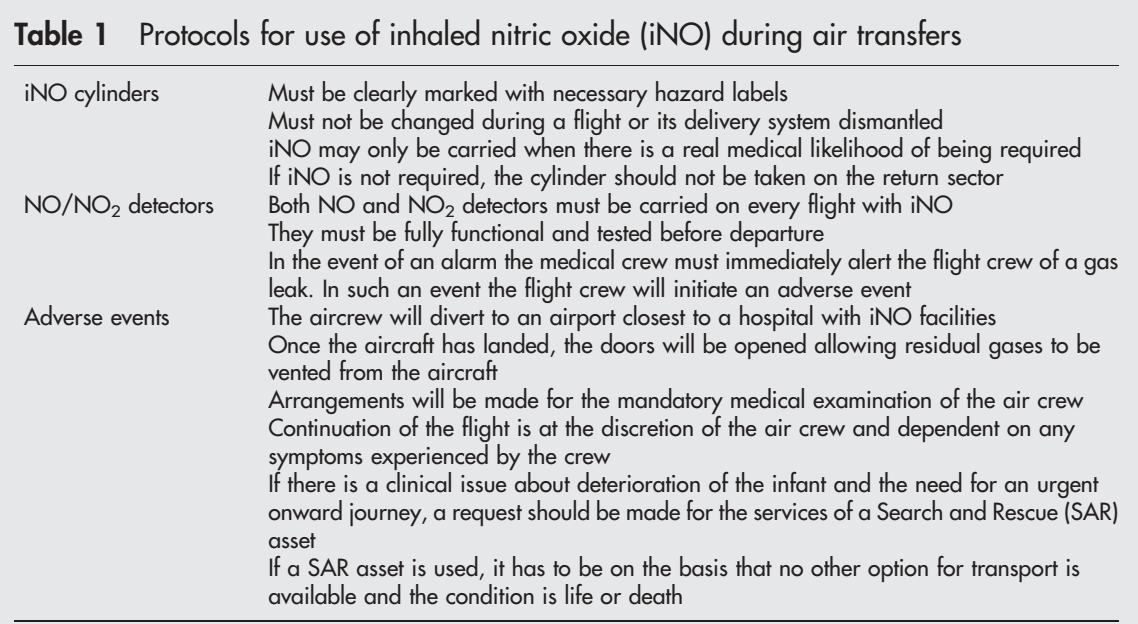

mattresses (Eden Medical) can help reduce the effects of vibration on both the baby and equipment.

Noise of up to $125 \mathrm{db}$ is experienced during take off and landing in the Beech King Air. In conjunction with turbulence, this can make diagnostic and clinical procedures difficult. The infant therefore should be stabilised and have all predictable procedures performed before transfer. Extra infusions or drugs that may be required during the journey should be prepared before flight departure. Visual rather than audio alarms should be used where possible.

The long term effects of exposure of the newborn infant to excessive sound remains unclear. ${ }^{10} 11$ Ear defenders for the infant should be considered, although Minimuffs (Natus Medical, San Carlos, California, USA) only reduced noise levels by $7 \mathrm{db}$. We are currently undertaking some work on the use of active noise reduction systems in this situation.

\section{TO VENTILATE OR NOT TO VENTILATE?}

Our guidelines indicate that if you consider there is a chance that mechanical respiratory support may be necessary, intubation and stable gas exchange should be achieved before departure. This is not usually a difficult decision. We intubate all infants who are on nasal continuous positive airway pressure and require air transport. Animated discussion usually takes place when the need arises to transport an infant requiring prostaglandin infusion to maintain patency of the arterial duct. It is recognised that there may be cardiovascular downsides to ventilating an infant with hypoplastic left heart syndrome. However, it is possible that apnoea may occur during the flight or loading/unloading with severe clinical deterioration. ${ }^{12}$ In these circumstances, physical space and the environment may not be optimal to allow an emergency airway management procedure to take place. The chance of apnoea increases with higher prostaglandin doses. Short duration of therapy may give inadequate time to assess response, and prostaglandin related hyperthermia may be associated with greater apnoea risk. Our current policy is to ventilate all infants receiving prostaglandins if air transfer is required. Protocols, however, do vary. The New South Wales team, one of the most experienced in this area, ventilate all infants requiring more than $20 \mathrm{ng} / \mathrm{kg} / \mathrm{min}$ prostaglandin (Personal Communication, Dr Andrew Berry). This protocol requires to be revisited based on evidence rather than intuition.

\section{Inhaled nitric oxide (iNO) therapy}

To allow use of iNO during air transfers, several safety protocols were introduced, as there was understandable concern from aviation operators regarding the safety of this gas when carried in pressurised aircraft (table 1). These were developed between the aircraft operators, the Scottish Air Ambulance Service, and clinicians. If a transport team wishes to introduce iNO during air transfers, they should contact the flight operations inspector for the aviation carrier, who will then determine from EASA whether all directives under the Dangerous Goods Convention (Class 2 Division 2.3) have been satisfied. Procedures for the use of iNO in flight will be specific both to the aviation operator and the aircraft type and depend on aircraft ventilation positioning, frequency of air changes, and volume of both the dispersant gas and the containment area. The protocols developed (table 1) reflect the inherent dangers of using iNO in flight. The cylinder/ regulator assembly is the part most vulnerable to damage and subsequent leak of gas. This has to be approved by the aviation operators as having satisfied EASA standards. Environmental monitoring must take place continuously as well as a periodic check of cylinder contents. There is no place for the use of iNO in an unplanned and uncertified manner. Aircrew should always be aware of the presence of cylinders of nitric oxide in their aircraft.

\section{STAFF ISSUES}

Staff who are involved in air transport should in the first instance be fully trained and experienced road transfer team members. In addition to this they must be aware of the clinical aspects of air physiology and have received appropriate training in transport by fixed wing and helicopter. This training must also include practical "dry runs" teamed with an experienced transport physician or nurse. ${ }^{13}$ They should not be prone to severe motion sickness.

Health and safety is of major importance. We offer all staff the opportunity to complete underwater survival training (Nutec, Aberdeen, Scotland, UK) and provide survival suits (Shark Group, Morpeth, Northumberland, UK), flame retardant flying suits, life jackets (Shark Group), and flight helmets (Headset Services Limited, Shoreham, Sussex, UK). Transport team members should never leave home without a credit card and cash. Essential overnight items are carried in case of the team being stranded. These situations are considerably eased for our team by the flexibility and help we receive from the SAS.

\section{CONCLUSION}

The technical aspects of developing an air transport system fit to fly in specific aircraft is challenging. For those intending to start from scratch, it is vital to understand that there are few 
shortcuts and much bureaucracy. A clear project leader should be identified at an early stage, and key members of the team should be enrolled who have a likelihood of being able to participate for the entire duration of the project. Commercial sensitivities should be dealt with as they arise, keeping clearly in mind that the project is health orientated and not for financial gain.

\section{ACKNOWLEDGEMENTS}

We acknowledge the tremendous partnership working with Paul Westaway, Emergency Services Manager at Bond Air Services, Bill Coutts, Isabel Worgan-Blake, and Andrew Marsden all of SAS. Also acknowledged is the sterling engineering work undertaken developing the new TIS by Paraid UK Ltd, and Medical Physics support from John McLaren and Francis Loch in Princess Royal Maternity.

\section{Authors' affiliations}

C H Skeoch, L Jackson, Princess Royal Maternity, Glasgow G31 2ER, Scotland, UK; Charles.Skeoch@northglasgow.scot.nhs.uk

A M Wilson, Queen Mother's Hospital, Glasgow G3 8SJ

P Booth, Aberdeen Maternity Hospital, Cornhill Road, Aberdeen AB25 2ZL, Scotland, UK

Competing interests: CHS and $U$ have received financial support from Paraid UK Ltd to attend a neonatal conference.

Consent for publication was obtained from the subjects shown in figs 2 and 3

\section{REFERENCES}

1 Harris BH, Belcher JW. Equipment and planning for neonatal air transport. Med Instrum 1982;16:253-5.

2 Miller C. The physiologic effects of air transport on the neonate. Neonatal Netw 1994;13:7-10.

3 Blumen IJ, Abernethy MK, Dunne MJ. Flight physiology. Clinical considerations. Crit Care Clin 1992;8:597-618.

4 Teitel D, Rudolph AM. Perinatal oxygen delivery and cardiac function. Adv Pediatr 1985:32:321-47.

5 Fenton AC, Leslie A, Skeoch CH. Optimising neonatal transfer. Arch Dis Child Fetal Neonatal Ed 2004;89:F215-19.

6 Bowman E, Doyle LW, Murton L, et al. Increased mortality of preterm infants transferred between tertiary perinatal centres. $B M$ 1988;297:1098-100.

7 Bowman ED, Roy RN. Control of temperature during newborn transport: an old problem with new difficulties. J Paediatr Child Health 1997;33:398-401.

8 L'Herault J, Petroff L, Jeffrey J. The effectiveness of a thermal mattress in stabilizing and maintaining body temperature during the transport of very low-birth weight newborns. Appl Nurs Res 2001;14:210-19.

9 Gajendragadkar G, Boyd JA, Potter DW, et al. Mechanical vibration in neonatal transport: a randomized study of different mattresses. J Perinatol 2000;20:307-10.

10 Buckland L, Austin N, Jackson A, et al. Excessive exposure of sick neonates to sound during transport. Arch Dis Child Fetal Neonatal Ed 2003;88:F513-16.

11 Campbell AN, Lightstone AD, Smith JM, et al. Mechanical vibration and sound levels experienced in neonatal transport. Am J Dis Child 1984;138:967-70.

12 Grubbs TC Jr, Kraft NL. Neonatal transport issues with prostaglandin E1 infusions. Air Med J 2002;21:8-12.

13 Leslie A, Stephenson T. Neonatal transfers by advanced neonatal nurse practitioners and paediatric registrars. Arch Dis Child Fetal Neonatal Ed 2003;88:F509-12. 